\title{
MiR-26a regulates vascular smooth muscle cell calcification in vitro through targeting CTGF
}

\author{
Wu W, Shang YQ, Dai SL, Yi F, Wang XC \\ Department of Cardiothoracic Surgery, The Central Hospital of Wuhan, Tongji Medical College Huazhong \\ University of Science and Technology,Wuhan Hubei, China. shangyuqiang@mail.com
}

\begin{abstract}
Vascular calcification is one of the most important factors for high morbidity and mortality from cardiovascular and cerebrovascular diseases. The aim of this study is to investigate the effect and mechanism of miR-26a on vascular smooth muscle cell calcification. First, the VSMCs were induced by $\beta$-glycerol phosphate ( $\beta$-GP) for $7 \mathrm{~d}$ and $14 \mathrm{~d}$, and Alizarin Red S staining was performed to examine the mineralized nodule change; then real time RT-PCR and western blotting were performed to explore the expression of miR-26a, CTGF, OPG, RANKL and ALP in un-induced and $\beta$-GP-induced VSMCs; next, the VSMCs were transfected with miR-26a mimics, and Alizarin Red S staining was performed to examine the mineralized nodule change; finally, real time RT-PCR and western blotting were performed to explore the expression of miR-26a, CTGF, OPG, RANKL and ALP in un-transfected and miR-26a mimics transfected VSMCs. After $\beta$-GP treatment, $\beta$-GP promoted clear mineralized nodule changes, and miR-26a and OPG expression were significantly decreased and CTGF, RANKL and ALP expression were increased in VSMCs. Overexpression of miR-26a inhibited VSMCs calcification induced by $\beta$-GP, and regulated the expression of CTGF, OPG, RANKL and ALP. Our findings suggested that up-regulation of miR-26a before $\beta$-GP treatment inhibits VSMCs calcification through targeting CTGF (Fig. 4, Ref. 18). Text in PDF www.elis.sk.

KEY WORDS: MiR-26a, VSMCs, $\beta$-GP, CTGF signaling, calcification.
\end{abstract}

\section{Introduction}

Arterial calcification refers to the pathological process of calcium deposition in the arterial wall tissue. As a result, the arterial elasticity and hemodynamics decreased due to the deposition of calcium phosphate. It is common in diabetes, end-stage renal disease and in the elderly, and is an independent predictor of cardiovascular events (1). According to the location of calcium salt depositions, arterial calcification is divided into inner calcification and middle calcification. Calcification can be induced by inflammatory factors and lipids, and often coexist with atherosclerosis, which plays an important role in the occurrence of cardiovascular events. Medial calcification can be present without atherosclerosis, may be induced by many factors such as hyperphosphatemia, hypercalcinemiac, uraemia, vascular dementia (VD), and is common in cardiovascular events, end-stage renal disease and diabetes $(2,3)$.

There are many similarities between the process of arterial calcification and bone formation, which are the processes of active regulation. Epidemiological studies have also suggested that

Department of Cardiothoracic Surgery, The Central Hospital of Wuhan, Tongji Medical College Huazhong University of Science and Technology, Wuhan Hubei, China

Address for correspondence: Y.Q. Shang, MD, Department of Cardiothoracic Surgery, The Central Hospital of Wuhan, No. 26 Shengli Street, Jiangan District, Wuhan Hubei 430014, China.

Acknowledgments: The present study was supported by the Health and Family Planning Commission of Wuhan Municipality. there is a relationship between vascular calcification and bone metabolism disorders. Vascular smooth muscle cells (VSMCs) transdifferentiation to osteoblast-like cells is the cytological basis and key link of the middle artery calcification.

By microarray analysis, microRNA-26a can regulate osteoclast formation induced by RANKL and the target gene is connective tissue growth factor (CTGF) (4). Other targets include regulating osteogenic differentiation of adipose tissue-derived stem cells modulated by the SMAD1 transcription factor (5), promoting muscle synthesis by regulating Ezh2 (6). Many studies have shown that CTGF plays an important role in the process of tissue fibrosis and wound repair, which is the downstream molecule secreted by TGF- $\beta 1$-activated cells. Overexpression of CTGF stimulates vascular smooth muscle cell growth and migration in vitro, to express more extracellular matrix proteins such as collagen type I and fibronectin (7). CTGF promotes osteoclastogenesis induced by RANKL (8). However, the role of miRNA-26a in the regulation of vascular calcification in CTGF is unknown.

In this study, we identified that $\beta$-GP induces calcification of VSMCs, and examined the CTGF, OPG, RANKL and ALP expression levels and functional role of miR-26a in VSMCs.

\section{Material and methods}

\section{Cell culture and treatment}

Vascular smooth muscle cells (VSMCs) were obtained from the American Type Culture Collection (ATCC, USA) and grown in 
Od

A

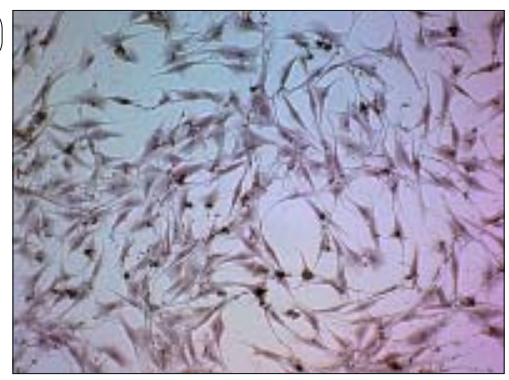

B

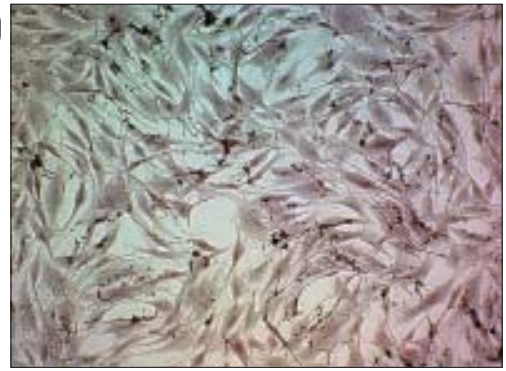

$7 d$
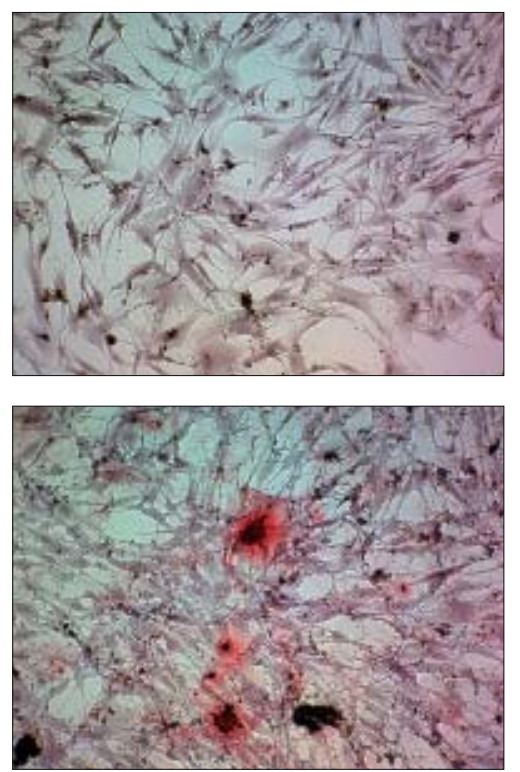

$14 d$
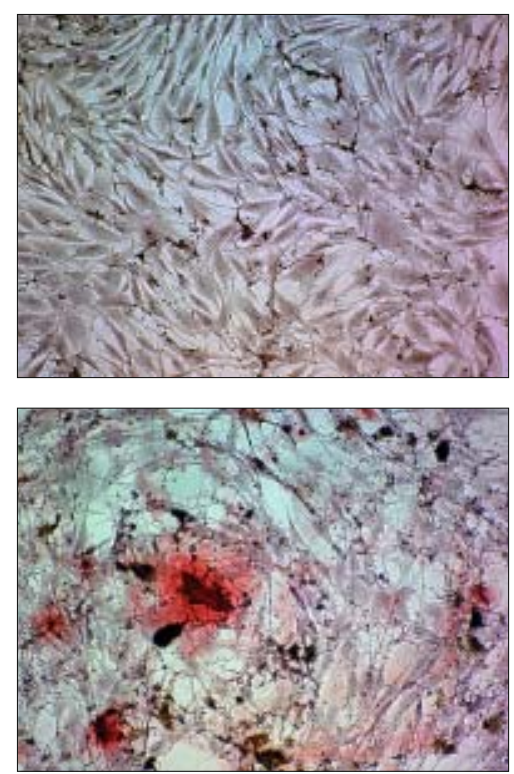

Fig. 1. $\beta$-GP induced VSMCs calcification. Alizarin Red S staining showed obvious calcium deposition in VSMCs incubated with $\beta$-GP media (B) compared with those with control media (A) for $7 \mathrm{~d}$ and $14 \mathrm{~d}$.

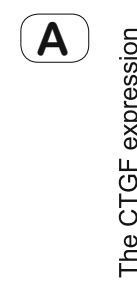
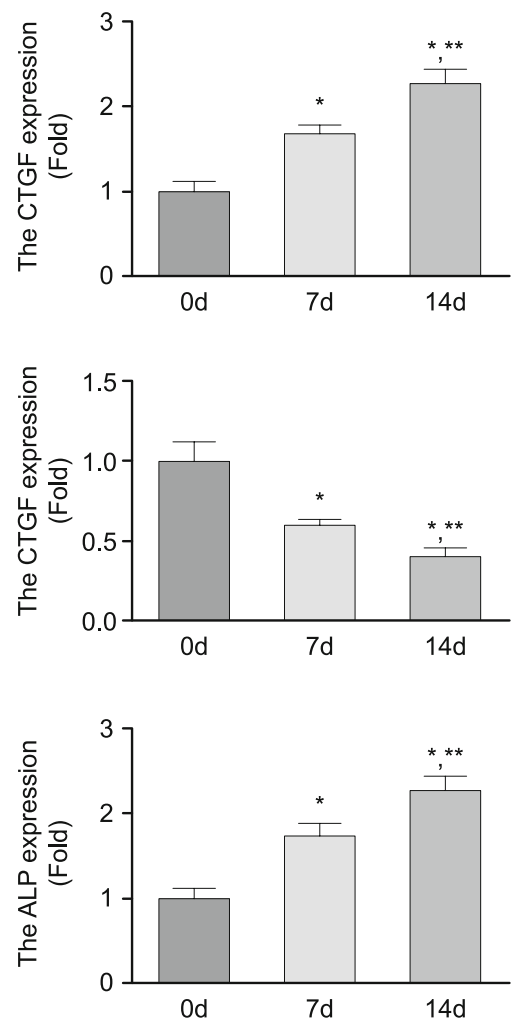
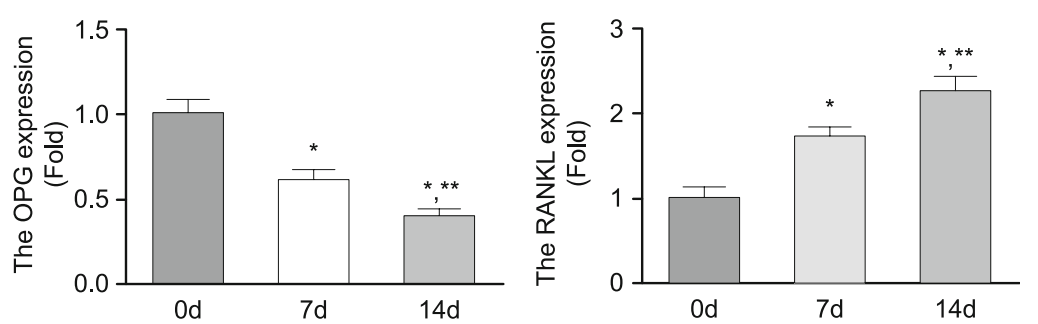

B

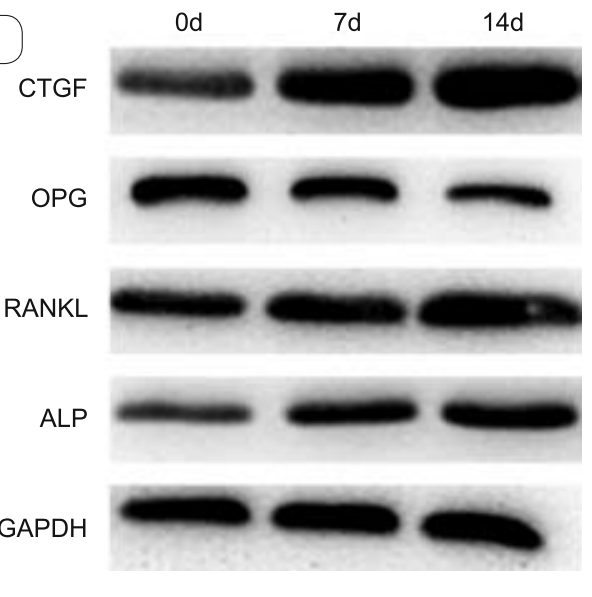

Fig. 2. Effects of $\beta$-GP on CTGF, OPG, RANKL and ALP expression. After VSMCs treated with $10.0 \mathrm{mmol} / \mathrm{l}$-GP for 7 days and 14 days, (A) RT-PCR and (B) Western blotting were measured. $\beta$-GP increased mRNA and protein levels of CTGF, RANKL and ALP, but decreased mRNA and protein levels of miR-26a and OPG (A); ${ }^{*} \mathrm{p}<0.05,{ }^{* *} \mathrm{p}<0.01$. 
RPMI-1640 Medium (GIBCO, Carlsbad, CA, USA) supplemented with $10 \%$ fetal bovine serum (FBS, GIBCO). For high phosphorus treatment, cells were incubated with $10 \mathrm{mmol} / \mathrm{L} \beta$-glycerol phosphate $(\beta-\mathrm{GP})$. After 7 and 14 days of the treatment, cells were harvested for real-time RT-PCR or Western blotting. MiR-26a mimics was transient transfected using Lipofectamine 2000 according to the instructions by the manufacturer (Invitrogen).

\section{Alizarin Red S staining}

For Alizarin Red S staining, the treated cells were incubated with $95 \%$ ethyl alcohol for $1 \mathrm{~h}$ at room temperature, then incubated with 1\% Alizarin Red S solution (Sigma-Aldrich) for $30 \mathrm{~min}$ at $37^{\circ} \mathrm{C}$. The staining results of mineralized nodules were observed under microscope.

\section{Real-time PCR (RT-PCR)}

Real-time PCR was performed as previously described (9). The primers were as follows: CTGF - forward 5'- CAG CAT GGA CGT TCG TCT G -3' and reverse 5'- AAC CAC GGT TTG GTC CTT GG -3'; OPG - forward 5'- GCG CTC GTG TTT CTG GAC A -3' and reverse 5'- AGT ATA GAC ACT CGT CAC TGG TG -3'; RANKL - forward 5'- CAA CAT ATC GTT GGA TCA CAG CA -3' and reverse 5' - GAC AGA CTC ACT TTA TGG GAA CC -3'; ALP - forward 5'- ACT GGG GCC TGA GAT ACC C-3' and reverse 5'- TCG TGT TGC ACT GGT TAA AGC -3';GAPDH - forward 5'-CGG AGT CAA CGG ATT TGG TCG TAT-3' and reverse 5'-AGC CTT CTC CAT GGT GGT GAA GAC-3'.

\section{Western blotting}

Western blotting was performed as previously described (9). The following primary antibodies were used: human monoclonal anti-CTGF, anti-OPG, anti-RANKL, anti-ALP and anti-GAPDH (1:1000; Santa Cruz Biotechnology, USA).

\section{Statistical analysis}

All data are expressed as mean \pm SD. Statistical analysis was performed using SPSS software version 19.0 (SPSS, IBM Corporation, Armonk, NY USA). The Student's t-test and ANOVA were used for the statistical analysis. $\mathrm{p}<0.05$ was considered as significant difference.

\section{Results}

$\beta$-GP induces calcification of VSMCs

To characterize the effects of $\beta$-GP in inducing calcification of VSMCs, we assessed mineralized nodule changes after $7 \mathrm{~d}$ and $14 \mathrm{~d}$. The results of Alizarin Red S staining showed that $\beta$-GP promoted clear mineralized nodule changes (Fig. 1). These results indicate that $\beta$-GP induces calcium deposition in VSMCs.

$\beta$-GP affects miR-26a, CTGF, OPG, RANKL and ALP expression

To clarify the mechanisms underlying the effects of $\beta$-GP in VSMCs, we measured miR-26a, CTGF, OPG, RANKL and ALP expression by RT-PCR and western blotting. The results of RT-PCR showed that $\beta$-GP promoted the expression of CTGF, RANKL and ALP mRNA, but inhibited the expression of miR-26a and OPG mRNA (p $<0.057 \mathrm{~d}, \mathrm{p}<0.001$ 14d) (Fig. 2A). And the results of western blotting showed that the levels of CTGF, RANKL and ALP protein were increased significantly, but the levels of miR-26a and OPG protein were decreased ( $\mathrm{p}<0.057 \mathrm{~d}, \mathrm{p}<0.001$ 14d) (Fig. 2B). These results indicate that $\beta$-GP induces calcification of VSMCs.

\section{MiR-26a inhibits VSMCs calcification induced by $\beta$-GP}

To determine the effects of miR-26a on VSMCs calcification induced by $\beta$-GP, we transfected VSMCs with miR-26a mimics and control oligo, and then assessed mineralized nodule changes. The results of Alizarin Red S staining showed that $\beta$-GP inhibits clear mineralized nodule changes (Fig. 3). These results indicate that miR-26a inhibits calcium deposition in VSMCs induced by $\beta$-GP.

MiR-26a regulates CTGF, OPG, RANKL and ALP expressions in VSMCs induced by $\beta$-GP

To further elucidate the mechanisms underlying the effects of miR-26a in VSMCs induced by $\beta$-GP was determined by RT-PCR and western blotting. The results of RT-PCR showed that transient overexpression of miR-26a reduced the expression levels of CTGF and RANKL mRNA, but promoted the expression of OPG mRNA $(\mathrm{p}<0.05)$ (Fig. 4A). And the results of western blotting showed that the levels of CTGF and RANKL protein were decreased significantly, but the level of OPG protein was increased $(p<0.05)$ (Fig. $4 B)$. No significant difference in the expression of ALP protein was
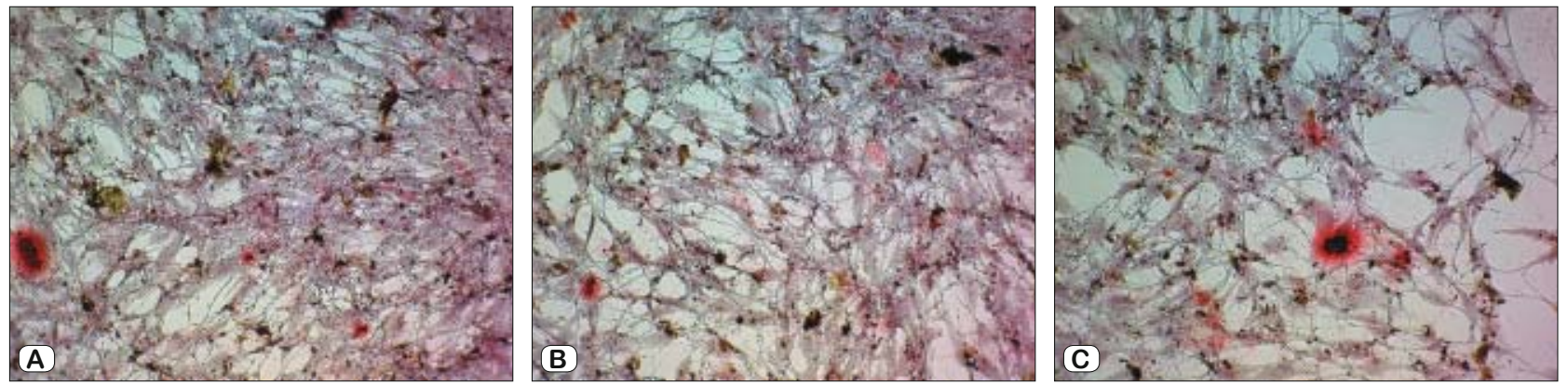

Fig. 3. Effects of miR-26a on VSMCs calcification induced by $\beta$-GP. Alizarin Red S staining showed obvious calcium deposition in cells transfected with miR-26a mimics (B) compared with negative control (A) and control oligo (C). 
499-503
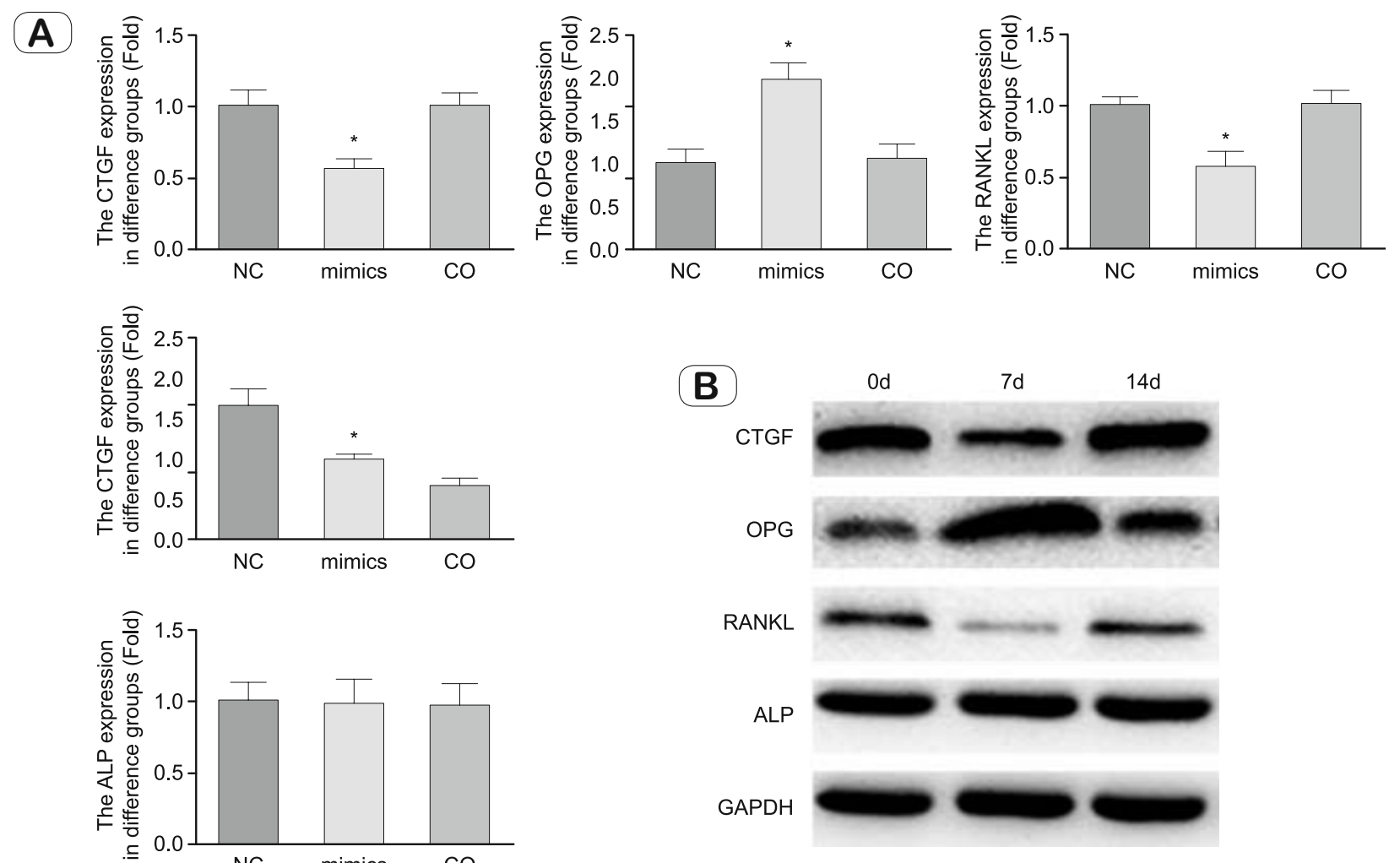

Fig. 4. Effects of miR-26a on CTGF, OPG, RANKL and ALP expression in VSMCs induced by $\beta$-GP. After VSMCs transfected with miR-26a mimics, (A) RT-PCR and (B) Western blotting were measured. MiR-26a decreased mRNA and protein levels of CTGF and RANKL, but increased mRNA and protein levels of OPG (A); ${ }^{*} \mathrm{p}<0.05$.

observed. These results indicate that transient overexpression of miR-26a decreased the calcification of VSMCs induced by $\beta$-GP.

\section{Discussion}

Vascular calcification is a common pathological manifestation of atherosclerosis, hypertension, diabetic vascular disease, vascular injury, chronic kidney disease and aging, mainly manifested as increased vascular wall stiffness, decreased compliance, myocardial ischemia, left ventricular hypertrophy and heart failure, leading to thrombosis and plaque rupture. Cardiovascular calcification is directly associated with increased cardiovascular and global morbidity and mortality. It represents an important part of the CKD-MBD (chronic kidney disease-mineral and bone disorders) complex and is a superior predictor of clinical outcomes in our patients $(1,10)$. Many recent studies have shown that high phosphate induces VSMCs calcification $(11,12)$. In this study, VSMCs were treated with $\beta$-GP, high phosphorus induced VSMCs trans-differentiation to osteoblast-like cells.

OPG/RANKL/RANK system is one of the important pathways to regulate bone metabolism. OPG (osteoprotegerin) is a member of the TNF-receptor superfamily, and an osteoblast-secreted decoy receptor that functions as a negative regulator of bone resorption. RANKL (Receptor Activator for Nuclear Factor- $\kappa \mathrm{B}$
Ligand), also known as TNF-related activation-induced cytokine (TRANCE), encodes a member of the tumor necrosis factor (TNF) cytokine family which is a ligand for osteoprotegerin and functions as a key factor for osteoclast differentiation and activation. CTGF is a mitogen that is secreted by vascular endothelial cells, which is one of the target genes of RANKL $(4,8,13-16)$. ALP is an alkaline phosphatase, a metalloenzyme that catalyzes the hydrolysis of phosphoric acid monoesters.

MicroRNAs (miRNA) are a class of non-coding single-stranded RNA molecules of about 22 nucleotides encoded by endogenous genes. They are involved in the regulation of a wide range of physiological processes. In recent years, many studies have shown that microRNAs play important roles in bone metabolism and vascular calcification. MiR-125b and miR-204 regulate transdifferentiation and calcification of vascular smooth muscle cells in a high-phosphate environment $(12,17)$. MiR-26a regulates extracellular matrix homeostasis in cartilage and RANKL-induced osteoclast formation $(4,18)$. In our study, VSMCs were transfected with miR-26a mimics, and then induced by $\beta$-GP. The results of Alizarin Red S staining displayed that miR-26a inhibits the calcification of $\beta$-GP-induced VSMCs. The results of RT-PCR and western blotting have shown that miR-26a promotes the level of OPG and inhibits the levels of CTGF and RANKL. Accordingly, we presume the possible mechanism involved is that overexpres- 
sion of miR-26a may restore the calcification of vascular smooth muscle cell calcification induced by high phosphorus.

In summary, we provide novel evidence that miR-26a is an important mediator which contributes to $\beta$-GP-induced calcification of VSMCs, providing a new target for therapeutic intervention of the diseases related to cardiovascular calcification.

\section{References}

1. Agarwal S, Bertazzo S. New paradigms in cardiovascular calcification. Comptes Rendus Chimie 2016; 19: 1605-1609.

2. Vervloet M, Cozzolino M. Vascular calcification in chronic kidney disease: different bricks in the wall? Kidney Int 2016.

3. Bostrom KI. Where do we stand on vascular calcification? Vasc Pharmacol 2016; 84: 8-14.

4. Kim K, Kim JH, Kim I, Lee J, Seong S, Park YW, Kim N. MicroRNA-26a regulates RANKL-induced osteoclast formation. Mol Cells 2015; 38: 75-80.

5. Luzi E, Marini F, Sala SC, Tognarini I, Galli G, Brandi ML. Osteogenic differentiation of human adipose tissue-derived stem cells is modulated by the miR-26a targeting of the SMAD1 transcription factor. J Bone Miner Res 2008; 23: 287-295.

6. Wong CF, Tellam RL. MicroRNA-26a targets the histone methyltransferase Enhancer of Zeste homolog 2 during myogenesis. J Biol Chem 2008; 283: 9836-9843.

7. Fan WH, Pech M, Karnovsky MJ. Connective tissue growth factor (CTGF) stimulates vascular smooth muscle cell growth, migration in vitro. Eur J Cell Biol 2000; 79: 915-923.

8. Nishida T, Emura K, Kubota S, Lyons KM, Takigawa M. CCN family $2 /$ connective tissue growth factor (CCN2/CTGF) promotes osteoclastogenesis via induction of and interaction with dendritic cell-specific transmembrane protein (DC-STAMP). J Bone Miner Res 2011; 26: 351-363.

9. Goettsch C, Rauner M, Pacyna N, Hempel U, Bornstein SR, Hofbauer LC. miR-125b regulates calcification of vascular smooth muscle cells. Am J Pathol 2011; 179: 1594-1600.
10. Bover J, Urena-Torres P, Gorriz JL, Lloret MJ, da Silva I, RuizGarcia C, Chang P, Rodriguez M, Ballarin J. Cardiovascular calcifications in chronic kidney disease: Potential therapeutic implications. Nefrologia 2016; 36: 597-608.

11. Lee KM, Kang HA, Park M, Lee HY, Choi HR, Yun CH, Oh JW, Kang HS. Interleukin-24 attenuates beta-glycerophosphate-induced calcification of vascular smooth muscle cells by inhibiting apoptosis, the expression of calcification and osteoblastic markers, and the Wnt/beta-catenin pathway. Biochem Biophys Res Commun 2012; 428: 50-55.

12. Wen P, Cao H, Fang L, Ye H, Zhou Y, Jiang L, Su W, Xu H, He W, Dai C, Yang J. miR-125b/Ets1 axis regulates transdifferentiation and calcification of vascular smooth muscle cells in a high-phosphate environment. Exp Cell Res 2014; 322: 302-312.

13. Harper E, Forde H, Davenport C, Rochfort KD, Smith D, Cummins PM. Vascular calcification in type-2 diabetes and cardiovascular disease: Integrative roles for OPG, RANKL and TRAIL. Vascul Pharmacol 2016; 82: 30-40.

14. Kagiya T, Nakamura S. Expression profiling of microRNAs in RAW264.7 cells treated with a combination of tumor necrosis factor alpha and RANKL during osteoclast differentiation. J Periodontal Res 2013; 48: 373-385.

15. Panizo S, Cardus A, Encinas M, Parisi E, Valcheva P, Lopez-Ongil S, Coll B, Fernandez E, Valdivielso JM. RANKL increases vascular smooth muscle cell calcification through a RANK-BMP4-dependent pathway. Circ Res 2009; 104: 1041-1048.

16. Wu M, Rementer C, Giachelli CM. Vascular calcification: an update on mechanisms and challenges in treatment. Calcif Tissue Int 2013; 93: 365-373.

17. Cui RR, Li SJ, Liu LJ, Yi L, Liang QH, Zhu X, Liu GY, Liu Y, Wu SS, Liao XB, Yuan LQ, Mao DA, Liao EY. MicroRNA-204 regulates vascular smooth muscle cell calcification in vitro and in vivo. Cardiovasc Res 2012; 96: 320-329.

18. Etich J, Holzer T, Pitzler L, Bluhm B, Brachvogel B. MiR-26a modulates extracellular matrix homeostasis in cartilage. Matrix Biol 2015; 43: $27-34$. 